\title{
Increased expression of herpesvirus entry mediator in 1,25-dihydroxyvitamin D3-treated mouse bone marrow-derived dendritic cells promotes the generation of $\mathrm{CD4}^{+} \mathrm{CD25}^{+} \mathrm{Foxp3}^{+}$regulatory $\mathrm{T}$ cells
}

\author{
YI HUANG ${ }^{1}$, YAN ZHAO ${ }^{2}$, XUEMEI RAN ${ }^{2}$ and CHANGZHENG WANG ${ }^{1}$ \\ ${ }^{1}$ Institute of Respiratory Diseases, Xinqiao Hospital, The Third Military Medical University, Chongqing 430037; \\ ${ }^{2}$ Department of Respiratory Medicine, Second Affiliated Hospital, Chongqing Medical University, \\ Chongqing 404000, P.R. China
}

Received June 17, 2013; Accepted December 11, 2013

DOI: $10.3892 / \mathrm{mmr} .2013 .1874$

\begin{abstract}
Dendritic cells (DCs) can initiate immune responses or induce immune tolerance. 1,25-dihydroxyvitamin $\mathrm{D} 3\left[1,25(\mathrm{OH})_{2} \mathrm{D} 3\right]$ is a secosteroid hormone that can induce tolerogenic dendritic cells favoring the induction of regulatory $\mathrm{T}$ cells (Treg). The present study revealed a tolerogenic effect of $1,25(\mathrm{OH})_{2} \mathrm{D} 3$ on the phenotype and function of ovalbumin (OVA)-activated mouse bone marrow-derived DCs. Three inhibitory molecules associated with tolerogenic DCs, programmed death-ligand-1 (PD-L1), PD-L2 and herpesvirus entry mediator (HVEM) and the expression of co-stimulatory molecules (CD80, CD86 and $\mathrm{CD} 40)$ on $1,25(\mathrm{OH})_{2} \mathrm{D} 3$-treated DCs were examined. The levels of interleukin (IL)-2, IL-6 and IL-10 secreted by $1,25(\mathrm{OH})_{2} \mathrm{D} 3$-treated DCs were analyzed. The capability of $1,25(\mathrm{OH})_{2} \mathrm{D} 3$-treated DCs to induce $\mathrm{CD} 4{ }^{+} \mathrm{CD} 25^{+} \mathrm{Foxp} 3^{+}$ Treg and the stimulation of allogeneic $\mathrm{CD}^{+} \mathrm{T}$-cell proliferation in mixed lymphocyte reaction (MLR) was studied. $1,25(\mathrm{OH})_{2} \mathrm{D} 3$-treated DCs induced up to 21.0 -fold upregulation of the HVEM expression and 4.1-fold enhancement of the HVEM expression upon activation with OVA [OVA-D3/immature (im)DCs]. PD-L1 was not affected by $1,25(\mathrm{OH})_{2} \mathrm{D} 3$-treated DCs and downregulated PD-L2 expression on $1,25(\mathrm{OH})_{2} \mathrm{D} 3$-treated DCs and OVA-D3/imDC. The expression of co-stimulatory molecules (CD80, CD86 and CD40) was downregulated in $1,25(\mathrm{OH})_{2} \mathrm{D} 3$-treated DCs and OVA-D3/imDC. Furthermore, 1,25(OH $)_{2}$ D3-treated DCs
\end{abstract}

Correspondence to: Professor Changzheng Wang, Institute of Respiratory Diseases, Xinqiao Hospital, Third Military Medical University, 183 Xinqiao Street, Shapingba, Chongqing 430037, P.R. China

E-mail: czwang@netease.com

Key words: herpesvirus entry mediator, regulatory T-cell, 1,25-dihydroxyvitamin D3, bone marrow derived dendritic cells secreted much higher levels of IL-10, however lower levels of IL-2 and IL-6 compared with the activated control DCs. Together with this pattern of cytokines, $1,25(\mathrm{OH})_{2} \mathrm{D} 3$-treated DCs exhibited low allogeneic CD4 ${ }^{+}$T-cell stimulatory activity and a higher number of $\mathrm{CD} 4{ }^{+} \mathrm{CD} 25^{+} \mathrm{Foxp}^{+}$cells in the MLR cultures but not in the activated control DCs. These findings indicate that $1,25(\mathrm{OH})_{2} \mathrm{D} 3$ possesses the immunosuppressive properties by upregulating the expression of the inhibitory molecules, HVEM, which may be therapeutically useful in controlling chronic immune and/or inflammatory diseases.

\section{Introduction}

The activated form of vitamin D, 1,25-dihydroxyvitamin D3 $\left[1,25(\mathrm{OH})_{2} \mathrm{D} 3\right]$ has, in addition to its central function in calcium and bone metabolism (1), important effects on the growth and differentiation of a number of cell types, and immunoregulatory properties. The biological effects of $1,25(\mathrm{OH})_{2} \mathrm{D} 3$ are mediated by the vitamin D receptor (VDR), a member of the superfamily of nuclear hormone receptors functioning as a ligand-activated transcription factor that binds to specific DNA sequence elements in vitamin D responsive genes and affects their rate of RNA polymerase II-mediated transcription (2). A number of studies have demonstrated an immunoregulatory effect for this steroid hormone (3-7). Antigen presenting cells (APCs), in particular DCs, express the VDR and are key targets of VDR agonists. Numerous studies have demonstrated that $1,25(\mathrm{OH})_{2} \mathrm{D} 3$ inhibits the differentiation and maturation of DCs (8-12). These studies have shown that $1,25(\mathrm{OH})_{2} \mathrm{D} 3$-treated DCs downregulated expression of the co-stimulatory molecules CD40, CD80, CD86 and MHC class II, decreased IL-12 and enhanced IL-10 production, resulting in decreased T-cell activation in vitro.

Besides the co-stimulatory molecules, inhibitory molecules are crucial to the tolerogenic capacity acquired by DCs. Members of the B7 family, programmed death ligand-1 (PD-L1) and PD-L2 have been found to be important in DC-mediated immune tolerance (13). In addition, PD-L1 signaling regulates the generation of $\mathrm{CD}^{+}{ }^{+} \mathrm{Foxp}^{+}$regulatory $\mathrm{T}$ cells (Treg) (14). 
Chen et al (15) found that PD-L1 and PD-L2 contribute to the poor stimulatory capacity of immature DCs (imDCs) via engagement of the inhibitory PD-1. However, PD-1 ligand blockade could not endow the same stimulatory capacity of imDCs as mature DCs (mDCs), which implied that other negative molecules could be involved in this process.

Herpesvirus entry mediator (HVEM), also known as tumor necrosis factor receptor superfamily, member 14 (TNFRSF14), is a member of the TNFR, is expressed by several types of cells, including T cells, B cells and DCs (16-18), can regulate the differentiation of T cells and DCs. HVEM can also regulate DC-mediated T-cell immune responses. Certain studies have demonstrated that $\mathrm{HVEM}^{-/}$mice were more susceptible to autoimmune diseases and APCs from $\mathrm{HVEM}^{-/-}$mice were more active in stimulating $\mathrm{T}$ cells compared with those from wild type (WT) mice (19). HVEM overexpression on APCs inhibited ovalbumin (OVA) peptide-dependent T-cell proliferation (20). HVEM overexpression on DCs produced a regulatory cytokine, IL-10, which had further effects on the induction of IL-10 producing $\mathrm{CD}^{+} \mathrm{T}$ cells (21). These findings supported the role of HVEM as an inhibitor molecule involved in DC-mediated immunological tolerance. However, the expression of HVEM on tolerogenic dendritic cells (tDCs) induced by VDR agonists remain incompletely characterized. Therefore, the expression of inhibitory molecules HVEM, PD-L1 and PD-L2 on 1,25(OH) 2 D3-treated mouse bone marrow-derived DCs was analyzed.

The data of the present study verified that the upregulated expression of the inhibitory molecule HVEM in $1,25(\mathrm{OH})_{2} \mathrm{D} 3$-treated DCs induced CD $4^{+} \mathrm{CD} 25^{+} \mathrm{Foxp}^{+}$Treg and arrested allogeneic $\mathrm{CD} 4^{+} \mathrm{T}$-cell proliferation. All these results further support the critical role of the inhibitory molecule HVEM in DC-mediated immune tolerance.

\section{Materials and methods}

Experimental animals. Female C57BL/6J and Balb/c mice were used at ages 6-8 weeks (Chongqing Experimental Animal Co., Chongqing, China). All the mice were bred under specific pathogen-free conditions. All the experiments were approved by an Ethics Committee (The Ethics Committee, Xinqiao Hospital, Third Military Medical University, Jiangyin, Jiangsu, China).

Preparation of DCs from mouse bone marrow. Murine bone marrow-derived DCs were prepared as described previously with minor modifications. Briefly, bone marrow mononuclear cells were prepared from C57BL/6 mouse tibia and femur suspensions by depletion of red cells and cultured at a density of $2 \times 10^{6}$ per well in six-well plates in RPMI-1640 medium supplemented with $10 \%$ fetal calf serum and $10 \mathrm{ng} / \mathrm{ml}$ recombinant murine granulocyte/macrophage colony-stimulating factor and $1 \mathrm{ng} / \mathrm{ml}$ recombinant murine IL-4 (22). Non-adherent cells were gently washed out on the third day of culture; the remaining loosely adherent clusters were cultured for a further 4 days as non-treated (NT) DCs. To produce the different subsets of DCs, imDCs were harvested on day 5 or 6 of culture. $1,25(\mathrm{OH})_{2} \mathrm{D} 3$-treated DCs (D3/imDCs) were generated by adding $10^{-8} 1,25(\mathrm{OH})_{2} \mathrm{D} 3$ on day 3 of NT-DCs culture and harvested on day 8 . OVA-D3/imDCs were generated by adding $100 \mu \mathrm{g} / \mathrm{ml}$ OVA on day 7 of the D3/imDCs culture for $24 \mathrm{~h}$ and harvested on day 8. Mature DCs (OVA/imDCs) was generated by adding $100 \mu \mathrm{g} / \mathrm{ml}$ OVA on day 7 of the NT-DCs culture for $24 \mathrm{~h}$ and harvested on day 8. DCs prepared in this manner

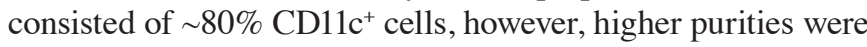
required thus the cells were sorted on $\mathrm{CD} 11 \mathrm{c}^{+}$by MACS beads (Miltenyi Biotec, Germany) to yield $~ 90 \%$ purity.

Flow cytometric analysis. DCs were triple-stained with APC-anti-CD11c and either PE-CD80,-MHC class II, -HVEM (CD270), -PDL1 (CD274) or FITC-CD86, -CD40, -PDL2 (CD273) for phenotypic analysis. The incidences of positive cells were determined by flow cytometry using a FACS Calibur (BD Biosciences, San Jose, CA, USA). Appropriately conjugated isotype-matched control antibodies were used as negative controls.

\section{Mixed lymphocyte reaction.}

DCs. Varying numbers of mitomycin C $(25 \mathrm{mg} / \mathrm{ml})$-treated DCs were seeded in triplicate in a flat-bottom 96-well plate (Corning, Tewksbury, MA, USA) for use as stimulator cells. T cells were prepared from spleens and isolated by $\mathrm{CD} 4^{+} \mathrm{T}$ cell magnetic microbeads (Miltenyi Biotec, Bergisch Gladbach, Germany), and the purity of the $\mathrm{CD}^{+} \mathrm{T}$ cells was $>95 \%$, according to the manufacturer's instructions. In total, $1 \times 10^{5} /$ well of $\mathrm{T}$ cells from BALB/c mice were added to the DC cultures, with the final MLR occurring in $200 \mu \mathrm{l}$ of RPMI1640 medium (Life Technologies, Grand Island, NY, USA) supplemented with $10 \%$ FCS (Life Technologies), $100 \mathrm{U} /$ $\mathrm{ml}$ penicillin and $100 \mu \mathrm{g} / \mathrm{ml}$ streptomycin (both from Life Technologies). The cells were cultured at $37^{\circ} \mathrm{C}$ in a humidified atmosphere of $5 \% \mathrm{CO}_{2}$ for 4 days, and pulsed with $1 \mu \mathrm{Ci}\left[{ }^{3} \mathrm{H}\right]$ thymidine (Amersham Pharmacia Biotech, Piscataway, NJ, USA) for the last $18 \mathrm{~h}$ of the culture. The cells were harvested onto glass fiber filters, and the radioactivity incorporated was quantitated using a Beckman liquid scintillation counter (Beckman Coulter, Miami, FL, USA). The results were expressed as the mean cpm of triplicate cultures \pm standard error of the mean.

In vitro differentiation assessment of $\mathrm{CD}^{+} \mathrm{CD}^{2} 5^{+} \mathrm{Foxp} 3^{+}$ Treg. Naïve CD $4{ }^{+} \mathrm{CD} 25-\mathrm{T}$ cells were purified from Balb/c spleen cells by magnetic-activated cell sorting (MACS) according to the manufacturer's instructions (Miltenyi Biotec). In total, $1 \times 10^{6}$ allogeneic $\mathrm{CD}^{+} \mathrm{CD} 25^{-} \mathrm{T}$ cells were cocultured with mitomycin C (25 mg/ml)-treated $1 \times 10^{5} \mathrm{OVA} / \mathrm{imDCs}$ and OVA-D3/imDCs for $96 \mathrm{~h}$. These $\mathrm{CD}^{+}{ }^{+} \mathrm{T}$ cells were then surface stained with FITC-anti-CD4 and PE-anti-CD25 mAbs and resuspended in Fix/Perm buffer (eBioscience, San Diego, CA, USA). Intracellular staining with PEcy5-anti-Foxp3 $\mathrm{mAb}$ (eBioscience) and estimation of the incidence of $\mathrm{CD} 4{ }^{+} \mathrm{CD} 25^{+} \mathrm{Foxp}^{+}$Treg was then determined by flow cytometry.

Enzyme-linked immunosorbent assay. Different subsets of DCs or DC coculture with naïve $\mathrm{CD} 4^{+} \mathrm{CD} 25^{-}-\mathrm{T}$ cells supernatants were stored at $-80^{\circ} \mathrm{C}$. The levels of IL-2, IL-6 and IL-10 were measured using ELISA kits (Cusabio Biotech Co., Ltd., Hubei, China), according to the manufacturer's instructions. The sensitivity limits for IL-2, IL-6 and IL-10 were $3.9 \mathrm{pg} / \mathrm{ml}$, 0.39 and $0.8 \mathrm{pg} / \mathrm{ml}$, respectively. 
A

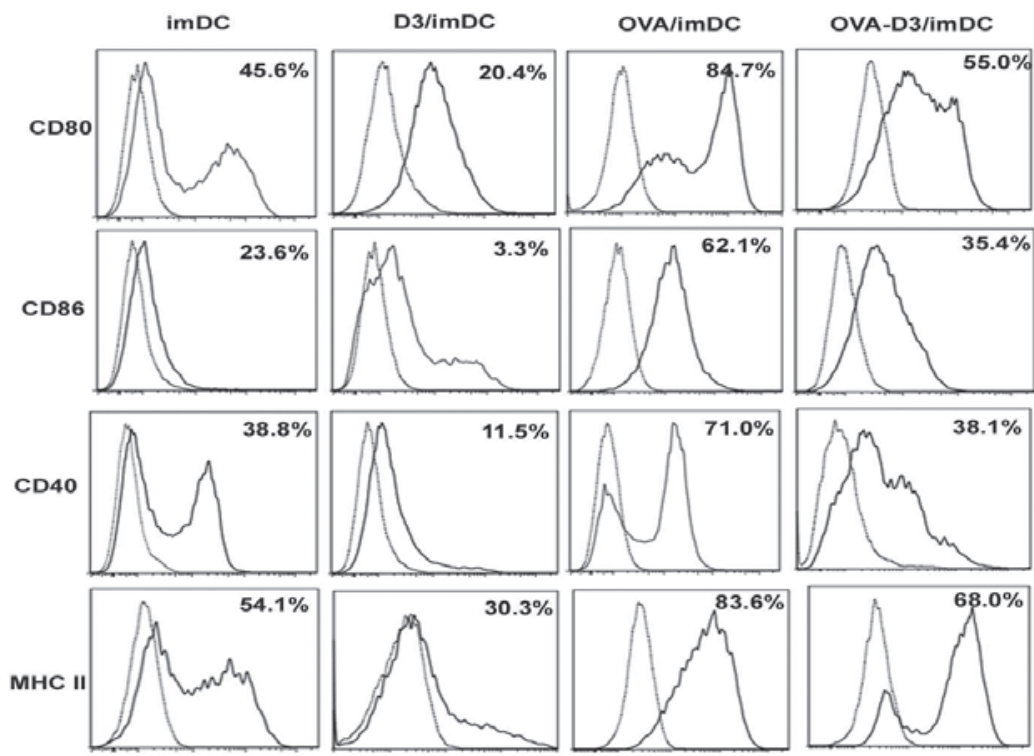

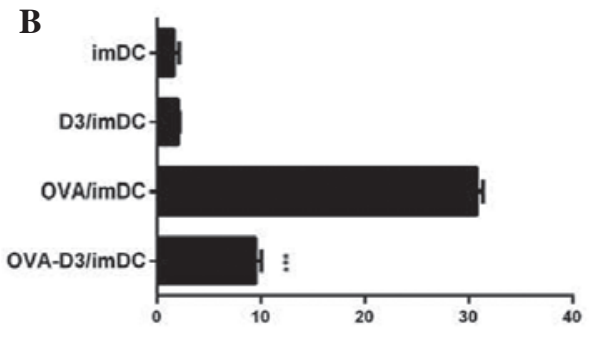

IL- 2 concentration(pg/ml)

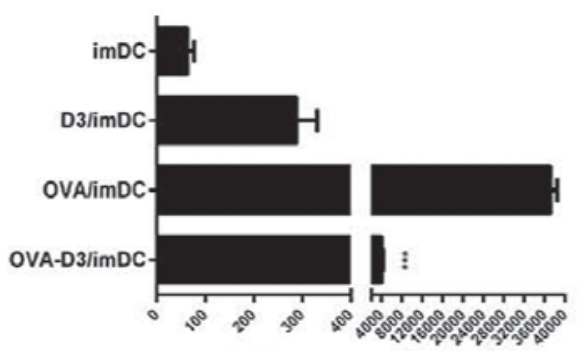

IL- 6 concentration(pg/ml)

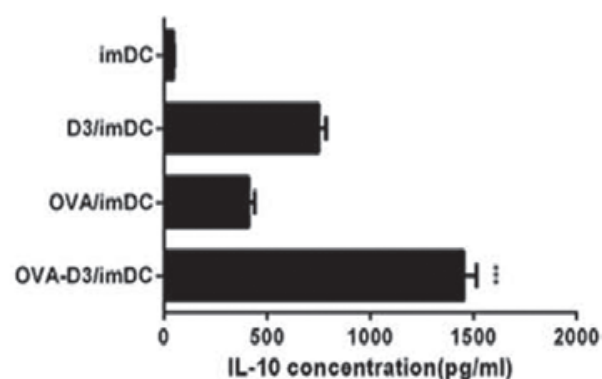

IL-10 concentration(pg/ml)

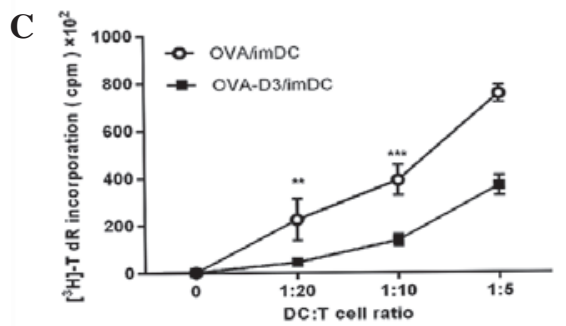

Figure 1. 1,25(OH) $)_{2}$ D3-treated bone marrow-derived dendritic cells exhibit tolerogenic features. (A) Expression of co-stimulatory molecules (CD80, CD86 and CD40) and MHC class II on DCs was measured by flow cytometry. Dashed lines represent isotype control mAb-staining. Solid lines represent surface molecule staining. Results are shown from a representative experiment out of 3-5 independent experiments performed. (B) IL-2, IL-6 and IL-10 levels. Results are shown from a representative experiment out of 3 independent experiments performed. (C) The allostimulatory capacities of D3/imDCs and OVA/imDCs were measured by MLR. ${ }^{* *} \mathrm{P}<0.01$ and ${ }^{* * *} \mathrm{P}<0.001$ indicate significant differences in comparison with controls. Results are shown from a representative experiment out of 3 independent experiments performed. MHC, major histocompatibility complex; DCs, dendritic cells; IL, interleukin; imDCs, immature DCs; OVA, ovalbumin; MLR, mixed lymphocyte reaction.

Statistical analysis. The data are presented as the mean \pm standard deviation. Statistical comparisons were performed using analysis of variance and the $\chi^{2}$ test. $\mathrm{P}<0.05$ was used to indicate a statistically significant difference. Data analyses were performed using SPSS version 13.0 (SPSS, Inc., Chicago, IL, USA).

\section{Results}

1,25(OH ${ }_{2}$ D3-treated bone marrow-derived dendritic cells exhibit features of tolerogenic DCs. The phenotype and T-cell stimulatory capacity of $1,25(\mathrm{OH})_{2} \mathrm{D} 3$-treated BMDCs activated by OVA (OVA-D3/imDCs) were analyzed and compared with those of mature DCs (OVA/imDCs). 1,25(OH) $)_{2} \mathrm{D} 3$-treated DCs (D3/imDCs) demonstrated immature phenotype, which downregulated the expression of the co-stimulatory molecules (CD80,CD86 and CD40) and MHC class II compared with those of immature DCs (all $\mathrm{P}<0.001)$. Upon activation by OVA, D3/imDCs demonstrated a semi-mature phenotype, with higher expression of co-stimulatory molecules CD86 and MHC class II compared with those of imDCs (all $\mathrm{P}<0.05$ ), but lower than OVA/imDCs (all $\mathrm{P}<0.001)$ (Fig. 1A). This indicates that $1,25(\mathrm{OH})_{2} \mathrm{D} 3$ inhibits DC maturation. Next, the cytokine production pattern of the OVA-D3/imDCs was examined. D3/imDCs secreted low levels of IL-2, IL-6 and intermediate levels of IL-10. Upon activation by OVA, D3/imDCs produced more 

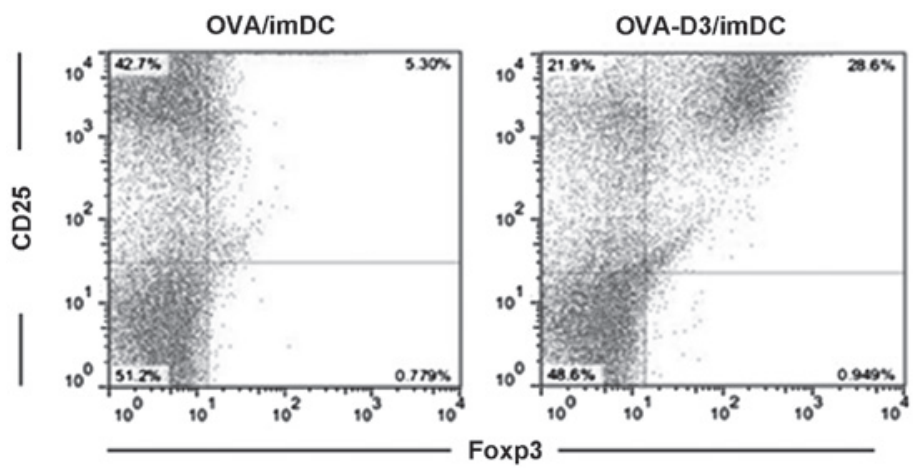

Figure 2. 1,25(OH) $)_{2}$ 3 -treated bone marrow-derived dendritic cells induce $\mathrm{CD} 4{ }^{+} \mathrm{CD} 25^{+} \mathrm{Foxp} 3^{+}$Treg. OVA/imDCs or OVA-D3/imDCs were co-cultured with $\mathrm{CD} 4^{+} \mathrm{CD} 25^{-} \mathrm{T}$ cells for $96 \mathrm{~h}$. The expression percentage of $\mathrm{CD} 4^{+} \mathrm{CD} 25^{+} \mathrm{Foxp} 3^{+}$(Treg) and CD4 ${ }^{+} \mathrm{CD} 25^{+} \mathrm{Foxp} 3^{-} \mathrm{T}$ (Teff) cells were detected by flow cytometry. Histograms gated on $\mathrm{CD} 4{ }^{+} \mathrm{CD} 25^{+} \mathrm{Foxp}^{+}$cells. The results are shown from a representative experiment out of 3 independent experiments performed. OVA, ovalbumin; imDCs, immature dendritic cells.

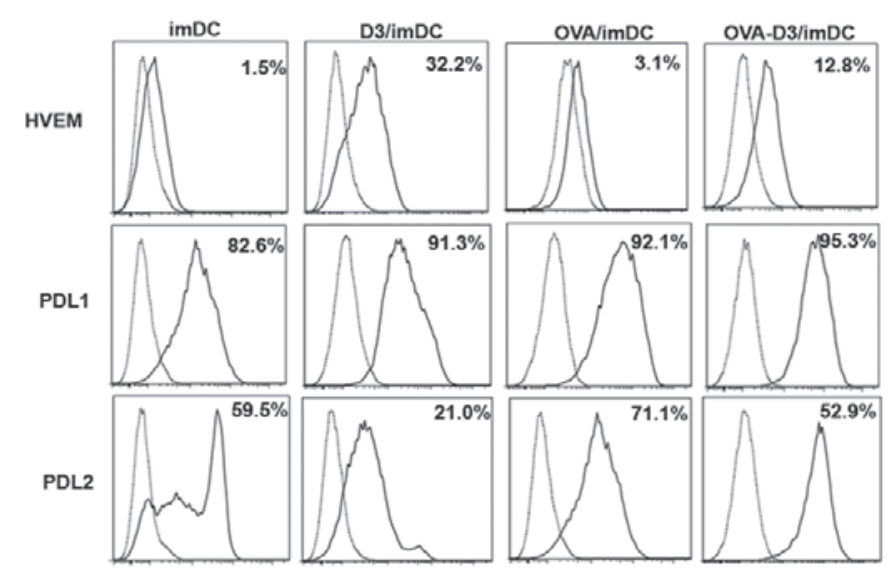

Figure 3. Expression of inhibitory molecules HVEM, PDL1 and PDL2 on 1, 25(OH $)_{2} \mathrm{D} 3$-treated bone marrow-derived dendritic cells. The expression of HVEM, PD-L1 and PD-L2 on imDCs, D3/imDCs, OVA/imDCs and OVA-D3/imDCs were measured by flow cytometry. Dashed lines represent isotype control $\mathrm{mAb}$ staining. Solid lines represent surface molecule staining. The results are shown from a representative experiment out of 3 independent experiments performed. HVEM, herpesvirus entry mediator; PDL, programmed death ligand; imDC, immature dendritic cells; mAB, monoclonal antibody.

IL-10 and less IL-2 and IL-6 compared with those produced by mature DCs (all $\mathrm{P}<0.001$ ) (Fig. 1B). These results indicate that $1,25(\mathrm{OH})_{2} \mathrm{D} 3$ inhibits the secretion of pro-inflammatory cytokines but promotes the secretion of anti-inflammatory cytokines. OVA-D3/imDCs exhibited a semi-mature phenotype and promoted secretion of anti-inflammatory cytokines. We speculated that OVA-D3/imDCs would be less capable of allogeneic $\mathrm{CD}^{+}{ }^{+} \mathrm{T}$-cell stimulation. Indeed, OVA-D3/imDCs demonstrated poor capacity to stimulate $\mathrm{CD} 4^{+} \mathrm{T}$-cell proliferation compared with OVA/imDCs at different DC:T cell ratio in a primary allogeneic MLR $(\mathrm{P}<0.01$ or 0.001 ; Fig. $1 \mathrm{C})$.

All these results support that $1,25(\mathrm{OH})_{2} \mathrm{D} 3$-treated dendritic cells possess tolerogenic phenotypes.

1,25(OH) ${ }_{2}$ D3-treated bone marrow-derived dendritic cells promote expansion of $\mathrm{CD}^{+} \mathrm{CD} 25^{+} \mathrm{Foxp} 3^{+}$Treg. $1,25(\mathrm{OH})_{2} \mathrm{D} 3$-treated BMDCs exhibit features of tolerogenic DCs. Next, whether the tolerogenic DCs may be correlated with specific interactions with $\mathrm{CD} 4^{+} \mathrm{CD} 25^{+} \mathrm{Foxp} 3^{+}$Treg was investigated. OVA-D3/imDCs or OVA/imDCs were cultured with $\mathrm{CD}^{+} \mathrm{CD} 25^{-} \mathrm{T}$ cells for $96 \mathrm{~h}$, and then the percentage of $\mathrm{CD} 4^{+} \mathrm{CD} 25^{+} \mathrm{Foxp}^{+}$Treg was analyzed by flow cytometry. As expected, a significantly high incidence of $\mathrm{CD} 4^{+} \mathrm{CD} 25^{+} \mathrm{Foxp} 3^{+}$
Treg and lower incidence of $\mathrm{CD} 4^{+} \mathrm{CD} 25^{+} \mathrm{Foxp} 3^{-}$effectorT cells (Teff) were detected from OVA-D3/imDC cultures compared with those of OVA/imDCs (Fig. 2). This indicates that $1,25(\mathrm{OH})_{2} \mathrm{D} 3$-treated dendritic cells promote expansion of $\mathrm{CD} 4^{+} \mathrm{CD} 25^{+} \mathrm{Foxp}^{+}$Treg and inhibit $\mathrm{CD} 4^{+} \mathrm{CD} 25^{+} \mathrm{Foxp} 3^{+}$Teff proliferation.

$1,25(\mathrm{OH})_{2} \mathrm{D} 3$ induces the expression of the inhibitory molecules HVEM and PD-L1 on bone marrow-derived dendritic cells. Inhibitory molecules HVEM, PD-L1, PD-L2 are known to negatively regulate T-cell responses $(19,23,24)$. Therefore, the expression of HVEM, PD-L1 and PDL2 on $1,25(\mathrm{OH})_{2} \mathrm{D} 3$-treated dendritic cells $(\mathrm{D} 3 / \mathrm{imDCs})$ were investigated by flow cytometric analysis. D3/imDCs induced up to 21.0-fold upregulation of HVEM expression and 1.1-fold upregulation of PD-L1 expression. OVA-D3/imDCs led to a 4.1-fold enhancement of HVEM expression and a slight 1.0-fold upregulation of PD-L1 expression (all $\mathrm{P}<0.001$ ). Conversely, D3/imDCs led to 2.8-fold downregulation of PD-L2 expression and OVA-D3/imDCs led to 1.5 -fold downregulation of PD-L2 expression, respectively (Fig. 3). These data implied that HVEM, but not PD-L2, is involved in tDCs induced by VDR agonists and promotes production of Treg. 


\section{Discussion}

In the present study, $1,25(\mathrm{OH})_{2} \mathrm{D} 3$-treated bone marrow-derived dendritic cells were verified to be tolerogenic DCs, which induced Treg generation and inhibited allogeneic CD4 ${ }^{+} \mathrm{T}$-cell proliferation. In addition, $1,25(\mathrm{OH})_{2} \mathrm{D} 3$ was observed to markedly upregulate the expression of the inhibitory molecule HVEM, which may be associated with tolerogenic DCs.

DCs not only initiate $\mathrm{T}$-cell responses but are also involved in the silencing of T-cell immune responses. Mature DCs induce differentiation of effector T cells (Th1, Th2) (25). Immature and semi-mature DCs appear to induce the differentiation of Treg and thus promote tolerance (26). The data of the present study confirmed the findings of previous studies (8-9) revealing that $1,25(\mathrm{OH})_{2} \mathrm{D} 3$-treated DCs significantly downregulated the co-stimulatory molecules CD86, CD80, CD40 and MHC II, which show an immature phenotype. Upon activation by OVA, 1,25(OH $)_{2}$ D3-treated DCs show a semi-mature phenotype, with higher expression of co-stimulatory molecule CD86, and MHC class II and decreased IL-2, IL-6 and enhanced IL-10 production. Furthermore, 1,25(OH) $)_{2} \mathrm{D} 3$-treated DCs demonstrate a poor capacity to stimulate $\mathrm{CD} 4^{+} \mathrm{T}$-cell proliferation in a primary MLR as previous studies have proved $(10,27,28)$. Indeed, $1,25(\mathrm{OH})_{2} \mathrm{D} 3$-treated $\mathrm{DCs}$ converted naïve $\mathrm{CD}^{+} \mathrm{T}$ cells into Foxp $3^{+}$Treg and suppressed allogeneic CD $4^{+} \mathrm{T}$-cell proliferation. All these results exhibited the tolerogenic properties of $1,25(\mathrm{OH})_{2} \mathrm{D} 3$-treated dendritic cells.

Besides the co-stimulatory molecules, it is observed that inhibitory molecules, including PD-L1 and PD-L2 (members of the B7 family) are involved in DC-mediated immune tolerance $(15,29)$. Unger et al (30) found PD-L1 was significantly upregulated on $1,25(\mathrm{OH})_{2} \mathrm{D} 3$-treated DCs and regulated the generation of Treg, as the blockade of PD-L1 eradicated the suppressive capacity of Treg. This indicates that the inhibitory molecule is critical for tDCs in the generation of Treg induced by $1,25(\mathrm{OH})_{2} \mathrm{D} 3$. However, the results of the present study identified that PD-L1 was hardly affected by $1,25(\mathrm{OH})_{2} \mathrm{D} 3$ and PD-L2 was downregulated on $1,25(\mathrm{OH})_{2} \mathrm{D} 3$-treated DCs, which implied that other negative molecules could be involved in this process.

HVEM, also known as TNFRSF14, is a member of the TNF family and their specific receptors (TNFR), which as an inhibitory molecule is also involved in DC-mediated immune tolerance. Studies have found that $\mathrm{HVEM}^{-/-}$mice were more susceptible to autoimmune diseases and APCs in $\mathrm{HVEM}^{-1-}$ mice were more active in stimulating $\mathrm{T}$ cells compared with WT mice (15). HVEM overexpression on APCs inhibits OVA peptide-dependent T-cell proliferation (20). HVEM overexpression on DC induced IL-10 producing CD4 ${ }^{+}-\mathrm{T}$ cells, and immune adoption of these DCs in vivo can protect against experimental autoimmune myocarditis (EAM) (21). However, very little is known regarding the role of HVEM in the induction of Treg by $1,25(\mathrm{OH})_{2} \mathrm{D} 3$-treated BMDCs.

The present study's observations that HVEM was significantly upregulated in $1,25(\mathrm{OH})_{2} \mathrm{D} 3$-treated DCs indicates that HVEM may be involved in the induction of Treg by $1,25(\mathrm{OH})_{2} \mathrm{D} 3$. However, it remains unclear what the exact underlying mechanism is. Kuipers et al (31) found that by triggering PD-L1 on DCs using soluble PD-1 immunoglobulin resulted in a decreased expression of the positive co-stimulatory molecules CD80, CD86 and CD40 and increased IL-10 production. An explanation may be reverse signaling by HVEM into DCs, resulting in a suppressive DC-phenotype. All these findings are based on the hypothesis that vitamin D regulates immune responses by controlling the expression of CYP27B1 and VDR (32-36).

In conclusion, increased HVEM on $1,25(\mathrm{OH})_{2} \mathrm{D} 3$-treated mouse-BMDCs induced $\mathrm{CD} 4{ }^{+} \mathrm{CD} 25^{+} \mathrm{Foxp} 3{ }^{+}$Treg and impaired allogeneic $\mathrm{CD}^{+}$T-lymphocyte proliferation, which further supported the important role of inhibitory molecules in DC-mediated immune tolerance.

\section{Acknowledgements}

This study was funded by the National Natural Science Foundation of China (nos. 30872341 and 81270075).

\section{References}

1. Casteels K, Bouillon R, Waer $M$ and Mathieu C: Immunomodulatory effects of 1,25-dihydroxyvitamin D3. Curr Opin Nephrol Hypertens 4: 313-318, 1995.

2. Carlberg C: Current understanding of the function of the nuclear vitamin D receptor in response to its natural and synthetic ligands. Recent Results Cancer Res 164: 29-42, 2003.

3. Griffin MD, Xing N and Kumar R: Vitamin D and its analogs as regulators of immune activation and antigen presentation. Annu Rev Nutr 23: 117-145, 2003.

4. Mathieu $\mathrm{C}$ and Adorini L: The coming of age of 1,25-dihydroxyvitamin D3 analogs as immunomodulatory agents. Trends Mol Med 8: 174-179, 2002.

5. Adorini L: Immunomodulatory effects of vitamin D receptor ligands in autoimmune diseases. Int Immunopharmacol 2: 1017-1028, 2002.

6. Adorini L: 1,25-Dihydroxyvitamin D3 analogs as potential therapies in transplantation. Curr Opin Investig Drugs 3: 1458-1463, 2002.

7. Deluca HF and Cantorna MT: Vitamin D: its role and uses in immunology. FASEB J 15: 2579-2585, 2001.

8. Penna $G$ and Adorini L: 1 Alpha,25-dihydroxyvitamin D3 inhibits differentiation, maturation, activation, and survival of dendritic cells leading to impaired alloreactive $\mathrm{T}$ cell activation. J Immunol 164: 2405-2411, 2000.

9. Piemonti L, Monti P, Sironi M, et al: Vitamin D3 affects differentiation, maturation, and function of human monocyte-derived dendritic cells. J Immunol 164: 4443-4451, 2000.

10. Griffin MD, Lutz WH, Phan VA, Bachman LA, McKean DJ and Kumar R: Potent inhibition of dendritic cell differentiation and maturation by vitamin D analogs. Biochem Biophys Res Commun 270: 701-708, 2000.

11. van Halteren AG, van Etten E, de Jong EC, Bouillon R, Roep BO and Mathieu C: Redirection of human autoreactive T-cells Upon interaction with dendritic cells modulated by TX527, an analog of 1,25 dihydroxyvitamin D(3). Diabetes 51: 2119-2125, 2002

12. Gauzzi MC, Purificato C, Donato K, et al: Suppressive effect of 1alpha,25-dihydroxyvitamin D3 on type I IFN-mediated monocyte differentiation into dendritic cells: impairment of functional activities and chemotaxis. J Immunol 174: 270-276, 2005.

13. Dai $\mathrm{H}$, Zhu H, Lei $\mathrm{P}$, et al: Programmed death-1 signaling is essential for the skin allograft protection by alternatively activated dendritic cell infusion in mice. Transplantation 88 : 864-873, 2009.

14. Wang L, Pino-Lagos K, de Vries VC, Guleria I, Sayegh MH and Noelle RJ: Programmed death 1 ligand signaling regulates the generation of adaptive Foxp3+CD4+ regulatory $\mathrm{T}$ cells. Proc Natl Acad Sci USA 105: 9331-9336, 2008.

15. Chen C, Qu QX, Huang JA, et al: Expression of programmed-death receptor ligands 1 and 2 may contribute to the poor stimulatory potential of murine immature dendritic cells. Immunobiology 212: 159-165, 2007. 
16. Kwon BS, Tan KB, Ni J, et al: A newly identified member of the tumor necrosis factor receptor superfamily with a wide tissue distribution and involvement in lymphocyte activation. J Biol Chem 272: 14272-14276, 1997.

17. Morel Y, Schiano de Colella JM, Harrop J, et al: Reciprocal expression of the TNF family receptor herpes virus entry mediator and its ligand LIGHT on activated T cells: LIGHT down-regulates its own receptor. J Immunol 165: 4397-4404, 2000.

18. Jung HW, La SJ, Kim JY, et al: High levels of soluble herpes virus entry mediator in sera of patients with allergic and autoimmune diseases. Exp Mol Med 35: 501-508, 2003.

19. Wang Y, Subudhi SK, Anders RA, et al: The role of herpesvirus entry mediator as a negative regulator of $\mathrm{T}$ cell-mediated responses. J Clin Invest 115: 711-717, 2005.

20. Sedy JR, Gavrieli M, Potter KG, et al: B and T lymphocyte attenuator regulates $\mathrm{T}$ cell activation through interaction with herpesvirus entry mediator. Nat Immunol 6: 90-98, 2005.

21. Cai G, Wang H, Qin Q, et al: Amelioration of myocarditis by HVEM-overexpressing dendritic cells through induction of IL-10-producing cells. Cardiovasc Res 84: 425-433, 2009.

22. Zhang M, Tang H, Guo Z, et al: Splenic stroma drives mature dendritic cells to differentiate into regulatory dendritic cells. Nat Immunol 5: 1124-1133, 2004.

23. Latchman Y, Wood CR, Chernova T, et al: PD-L2 is a second ligand for PD-1 and inhibits T cell activation. Nat Immunol 2 : 261-268, 2001.

24. Freeman GJ, Long AJ, Iwai Y, et al: Engagement of the PD-1 immunoinhibitory receptor by a novel B7 family member leads to negative regulation of lymphocyte activation. J Exp Med 192: 1027-1034, 2000.

25. Kaliński P, Hilkens CM, Wierenga EA and Kapsenberg ML: T-cell priming by type- 1 and type- 2 polarized dendritic cells: the concept of a third signal. Immunology Today 20: 561-567, 1999.

26. Lutz MB and Schuler G: Immature, semi-mature and fully mature dendritic cells: which signals induce tolerance or immunity? Trends Immunol 23: 445-449, 2002.

27. Anderson AE, Swan DJ, Sayers BL, et al: LPS activation is required for migratory activity and antigen presentation by tolerogenic dendritic cells. J Leukoc Biol 85: 243-250, 2009.
28. Alroy I, Towers TL and Freedman LP: Transcriptional repression of the interleukin-2 gene by vitamin D3: direct inhibition of NFATp/AP-1 complex formation by a nuclear hormone receptor. Mol Cell Biol 15: 5789-5799, 1995

29. Wölfle SJ, Strebovsky J, Bartz H, et al: PD-L1 expression on tolerogenic APCs is controlled by STAT-3. Eur J Immunol 41: 413-424, 2011.

30. Unger WW, Laban S, Kleijwegt FS, van der Slik AR and Roep BO: Induction of Treg by monocyte-derived DC modulated by vitamin D3 or dexamethasone: differential role for PD-L1. Eur J Immunol 39: 3147-3159, 2009.

31. Kuipers H, Muskens F, Willart M, et al: Contribution of the PD-1 ligands/PD-1 signaling pathway to dendritic cell-mediated CD4 ${ }^{+}$ T cell activation. Eur J Immunol 36: 2472-2482, 2006.

32. Sadeghi K, Wessner B, Laggner U, et al: Vitamin D3 down-regulates monocyte TLR expression and triggers hyporesponsiveness to pathogen-associated molecular patterns. Eur J Immunol 36: 361-370, 2006.

33. Hewison M, Freeman L, Hughes SV, et al: Differential regulation of vitamin D receptor and its ligand in human monocyte-derived dendritic cells. J Immunol 170: 5382-5390, 2003.

34. Dong X, Craig T, Xing N, et al: Direct transcriptional regulation of RelB by 1alpha,25-dihydroxyvitamin D3 and its analogs: physiologic and therapeutic implications for dendritic cell function. J Biol Chem 278: 49378-49385, 2003.

35. Griffin MD, Lutz W, Phan VA, Bachman LA, McKean DJ and Kumar R: Dendritic cell modulation by 1alpha,25 dihydroxyvitamin D3 and its analogs: a vitamin D receptor-dependent pathway that promotes a persistent state of immaturity in vitro and in vivo. Proc Natl Acad Sci USA 98: 6800-6805, 2001.

36. Szeles L,Keresztes G,Töröcsik D, et al: 1,25-dihydroxyvitamin D3 is an autonomous regulator of the transcriptional changes leading to a tolerogenic dendritic cell phenotype. J Immunol 182: 2074-2083, 2009 